Supporting Information:

\title{
Stable Aqueous Nanoparticle Film Assemblies with Covalent and Charged Polymer Linking Networks
}

\author{
Lesley E. Russell, Anne A. Galyean, Sherilyn M. Notte, and Michael C. Leopold* \\ Department of Chemistry, Gottwald Center for the Sciences, University of Richmond \\ Richmond, VA 23173
}

Contents:

- TEM Histogram Analysis and representative images

-Citrate stabilized nanoparticles (CS-NPs)

-Thioctic acid stabilized nanoparticles (TAS-NPs)

- UV-Vis spectra and TEM analysis of CS-NPs before/after ligand exchange with 15-crown-5 dodecanethiol.

- UV-Vis spectra corresponding to TEM imaging of Figure 1.

- UV-Vis spectra of dry monolayer and multi-layer films of CS-NPs and TAS-NPs (dithiol-linked) compared to solution species.

- Digital images of dithiol-linked and PL-linked TAS-NP film assemblies

- UV-Vis spectra of PL-linked TAS-NP films during stability testing in solutions of varying conditions ( $\mathrm{pH}$, temperature, and ionic strength)

- UV-Vis spectra of TAS-NP and CS-NP solutions with the addition of $\mathrm{NaCl}$ electrolyte. 

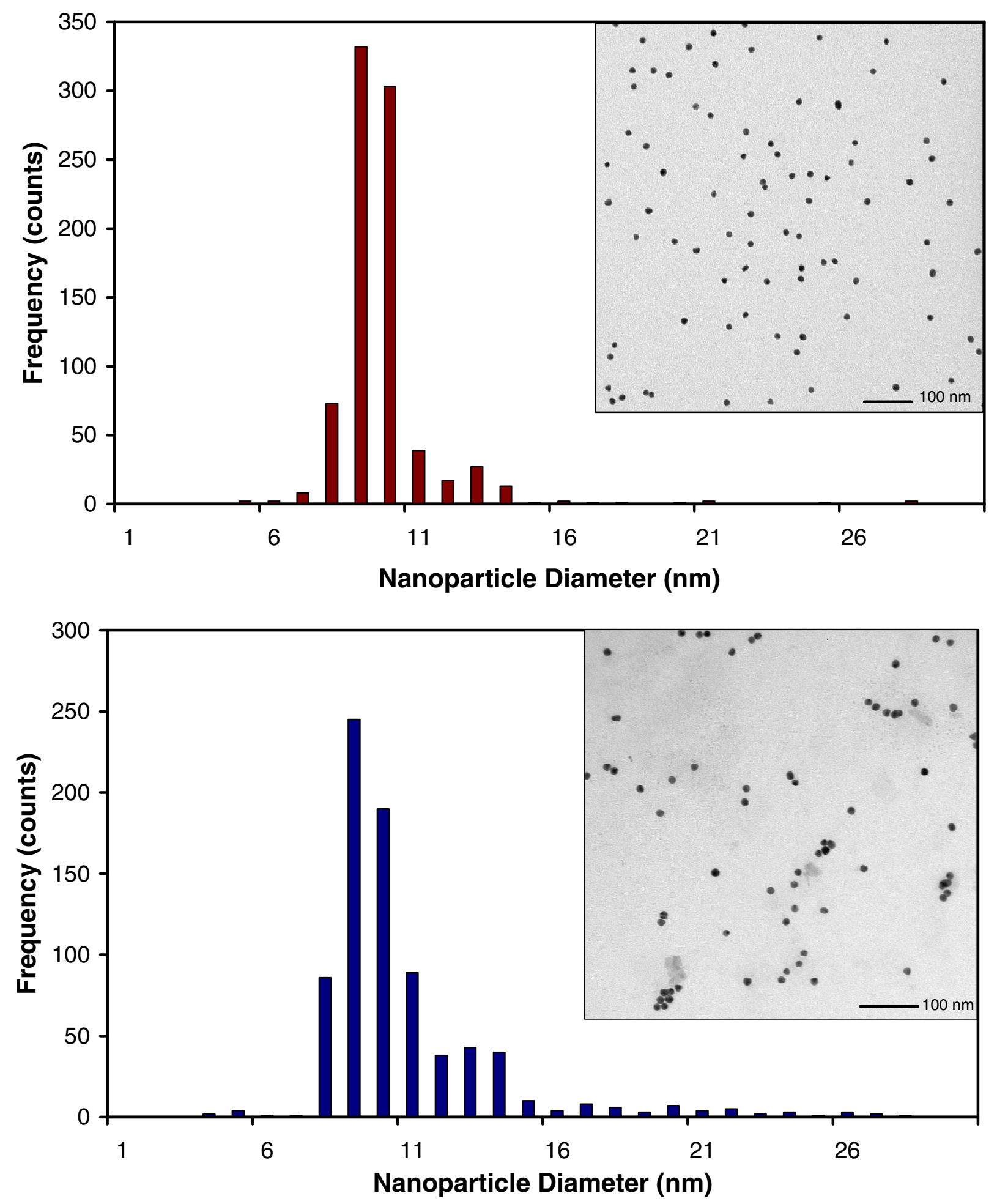

Figure SM-1. Core size histograms and typical images (Insets) from TEM analysis of CS-NPs (top) and TAS-NPs (bottom) immediately after synthesis. Histograms were generated using Image $\mathbf{J}$ image analysis program with greater than 800 counts. 

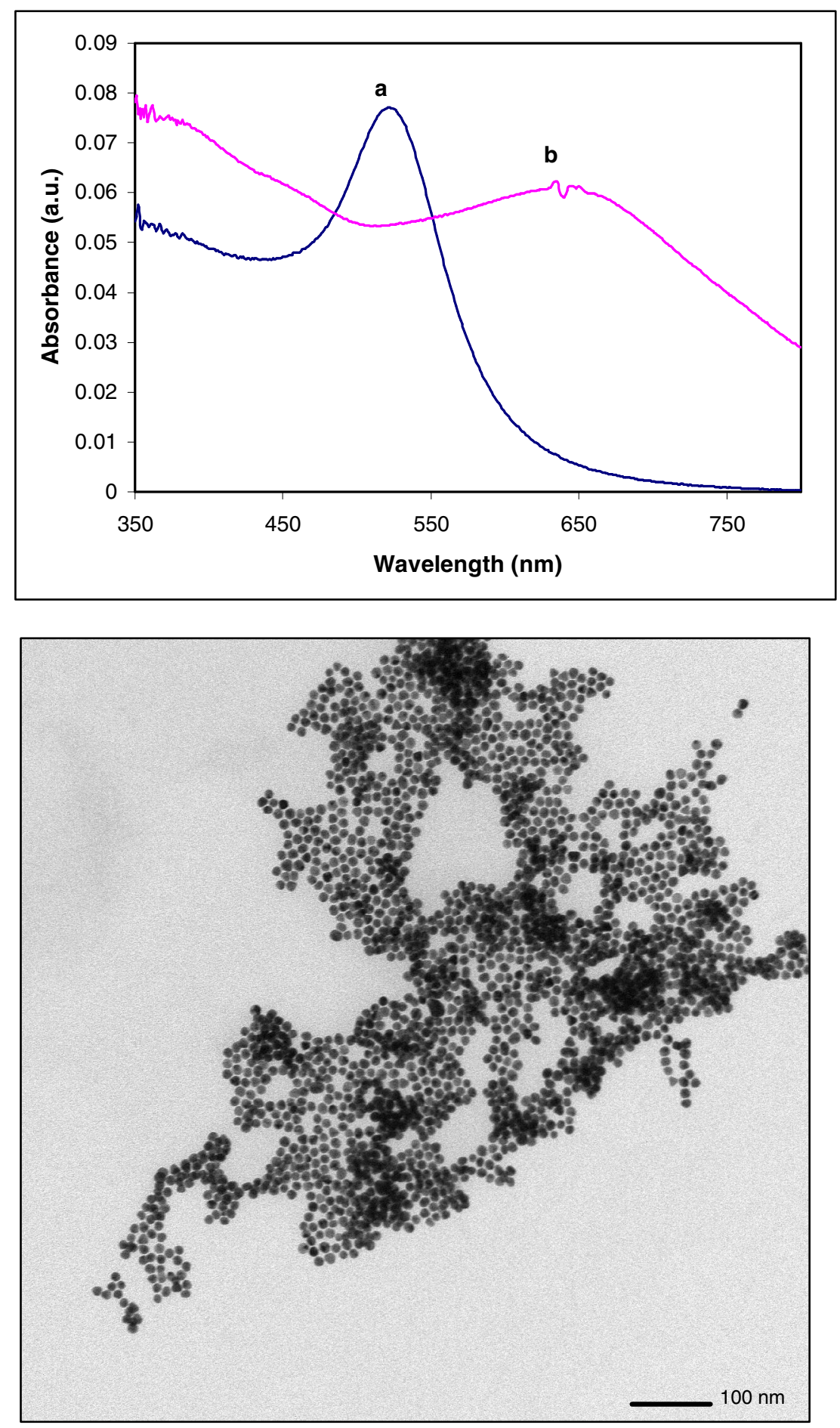

Figure SM-2. UV-Vis spectra of CS-NPs before (top, a) and after (top, b) exchange reaction with 15crown-5 dodecanethiol. Corresponding TEM image of CS-NPs after exchange (bottom). Massive aggregation/precipitation is visible in the reaction flask almost immediately during the exchange. ${ }^{\text {a }}$

${ }^{a}$ Lin, S.; Tsai, Y.; Chen, C.; Lin, C.; Chen, C. J. Phys. Chem. B 2004, 108, 2134-2139. 

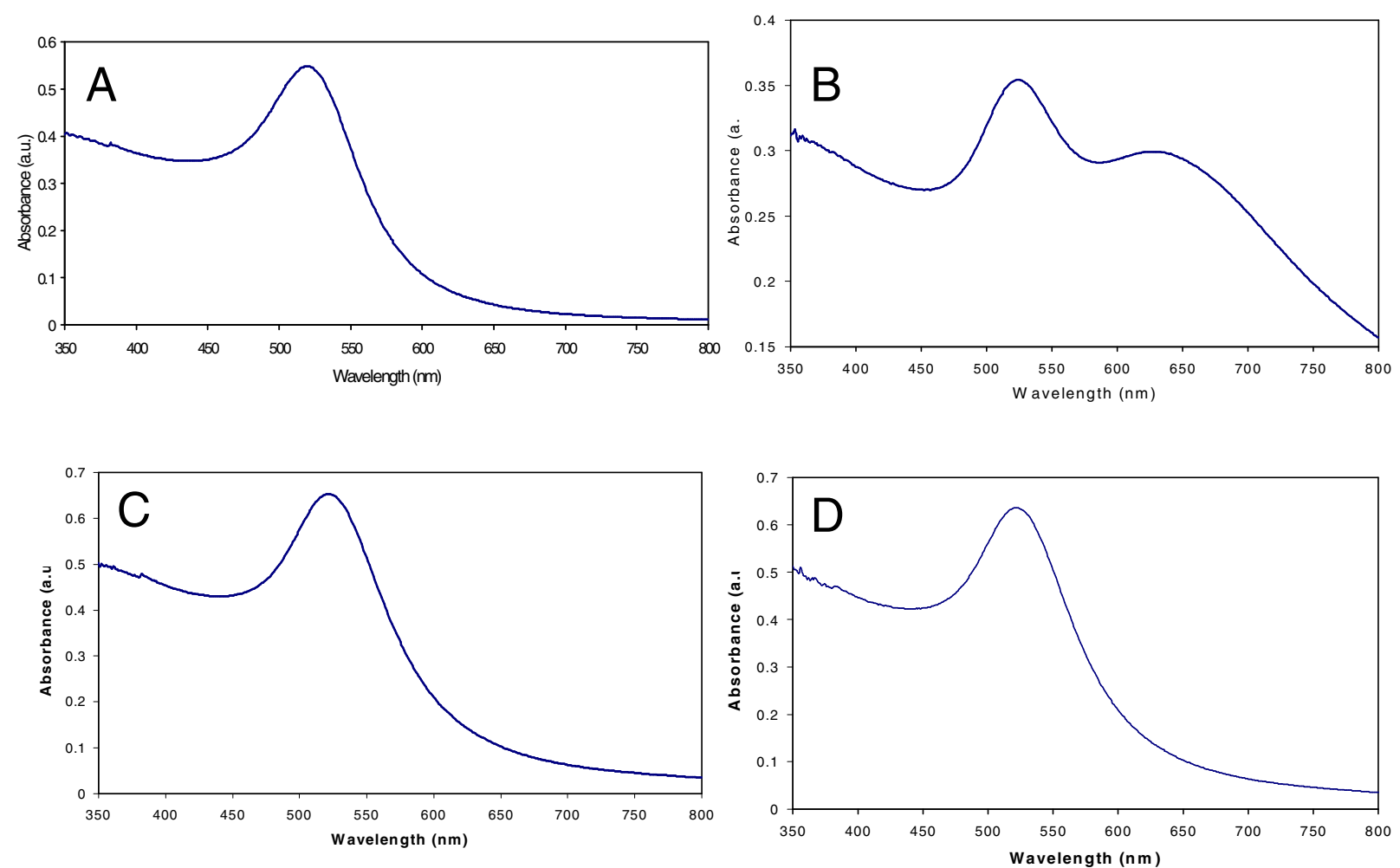

Figure SM-3. UV-Vis spectra corresponding to the TEM images shown in Figure 1. (top) Spectrum of CS-NPs immediately after synthesis (A) and after one week of storage (B). (bottom) TAS-NPs immediately after TA-stabilization (C) and after one week of storage (D). 

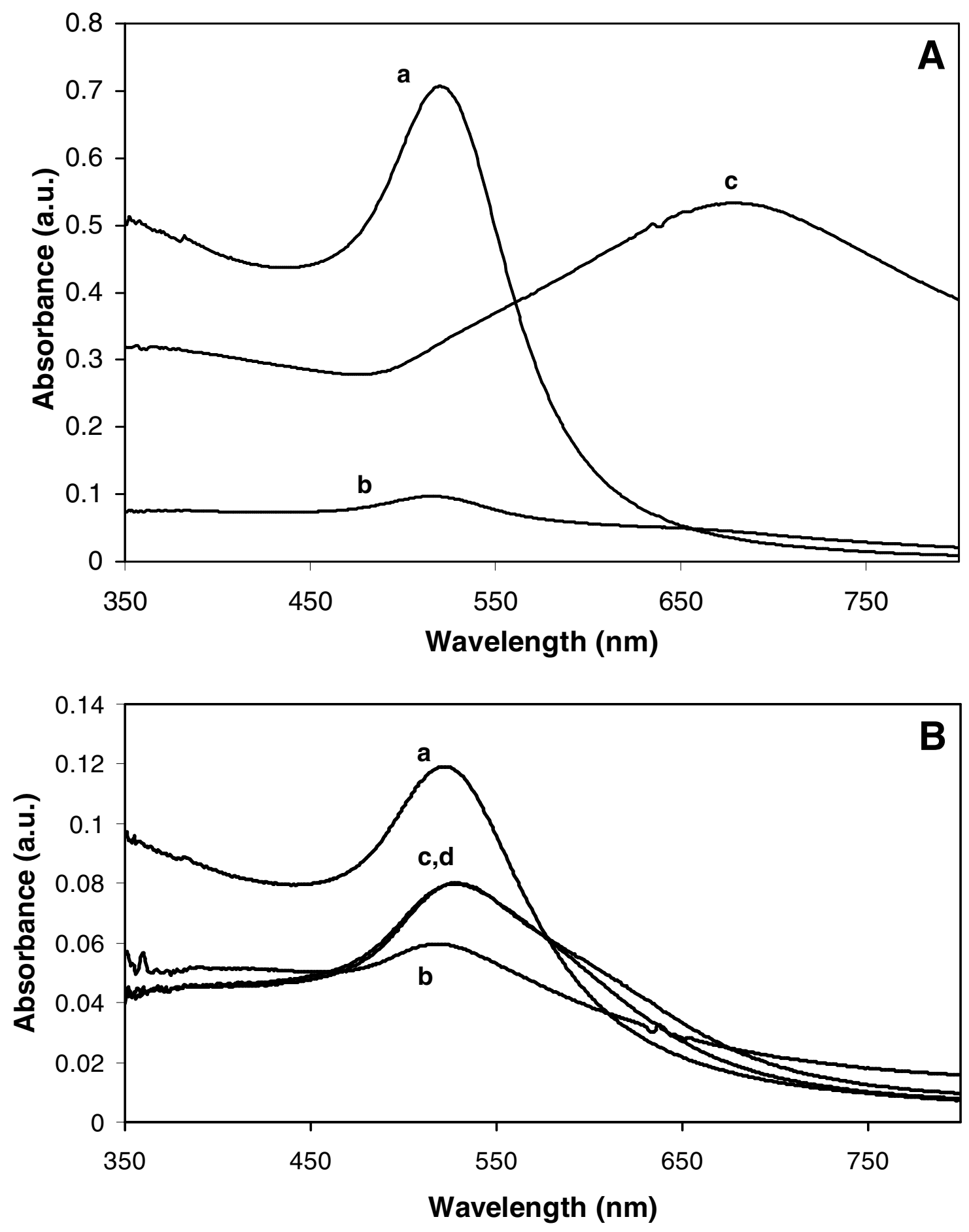

Figure SM-4. A) UV-Vis spectra of a CS-NP solution (a), a monolayer of CS-NPs on 3-APTMS modified glass in air (b), and a dithiol-linked multi-layer film of CS-NPs on 3-APTMS modified glass in air. (B) UV-Vis spectra of a TAS-NP solution (a), a monolayer of TAS-NPs on 3-APTMS modified glass in air (b), a dithiol-linked multi-layer film on 3-APTMS modified glass in air immediately after construction (c), and a dithiol-linked multi-layer film on 3-APTMS modified glass over 10 days after construction and stored in air (d). 


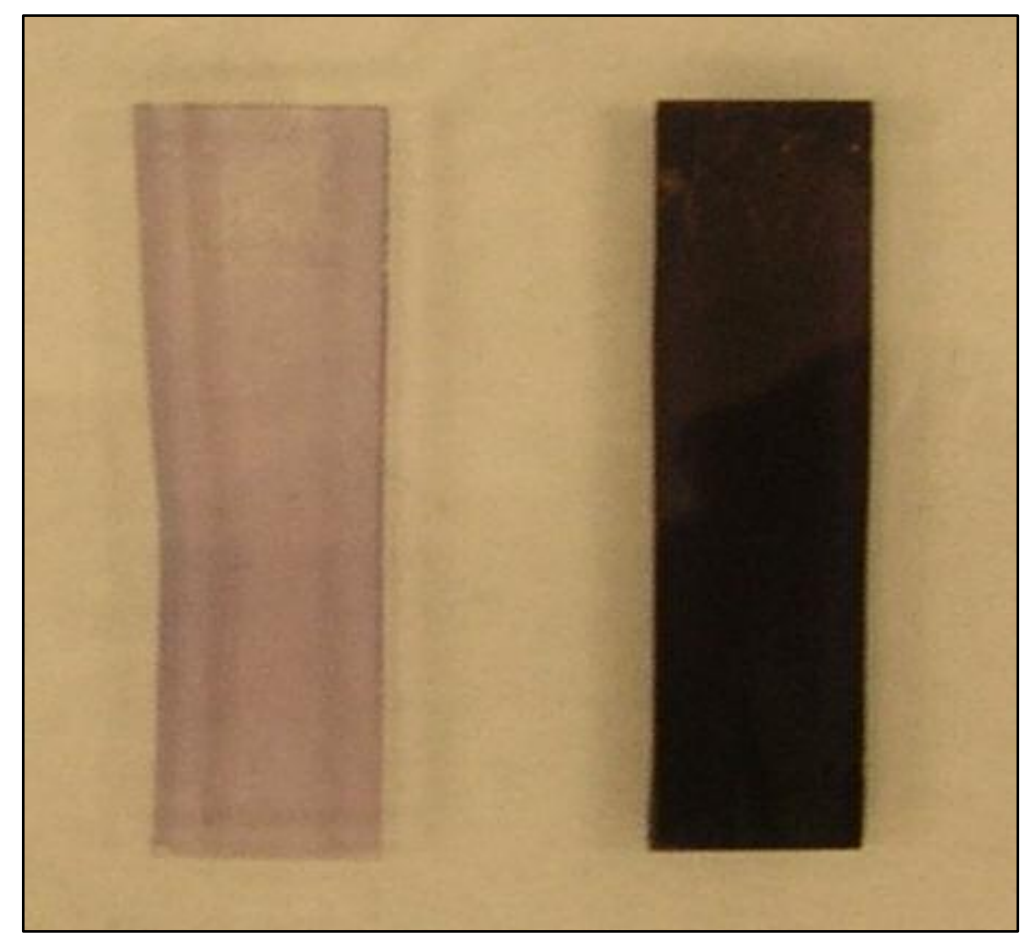

Figure SM-5. Digital images of dithiol-linked (left) and PL-linked (right) TAS-NP film assemblies after 8 dipping cycles. Images are provided to show the visually obvious difference in film thicknesses of the two types of films. 

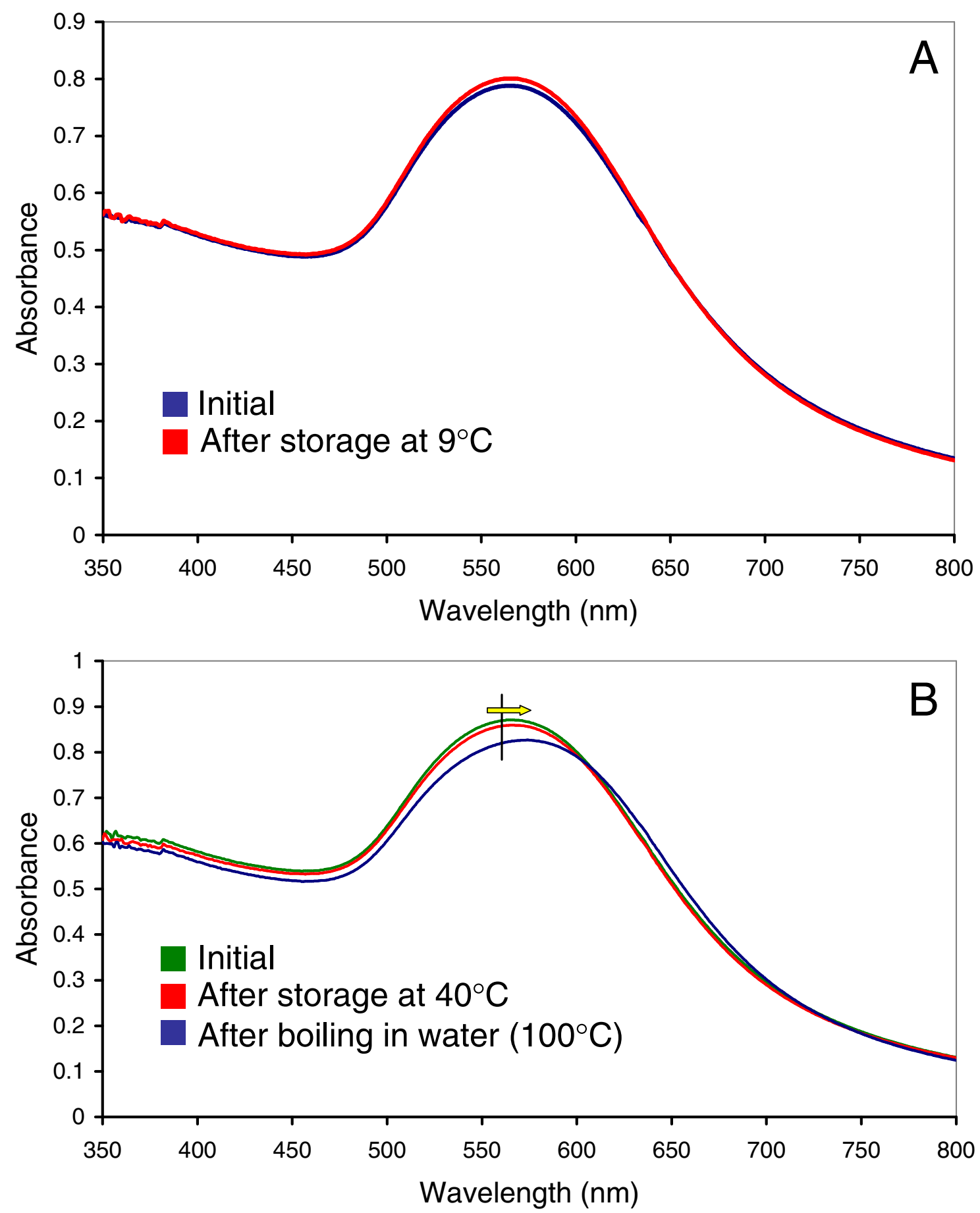

Figure SM-6. PL-linked TAS-NP films before/after exposure to water at (A) $9{ }^{\circ} \mathrm{C}$ and (B) $40^{\circ} \mathrm{C}, 100{ }^{\circ} \mathrm{C}$ for one hour. Note: Films were assembled as described in the Experimental Details, rinsed with purified water, exposed to solutions of different temperature for the designated time and rinsed with purified water before final immersion in purified water for UV-Vis spectroscopy. 


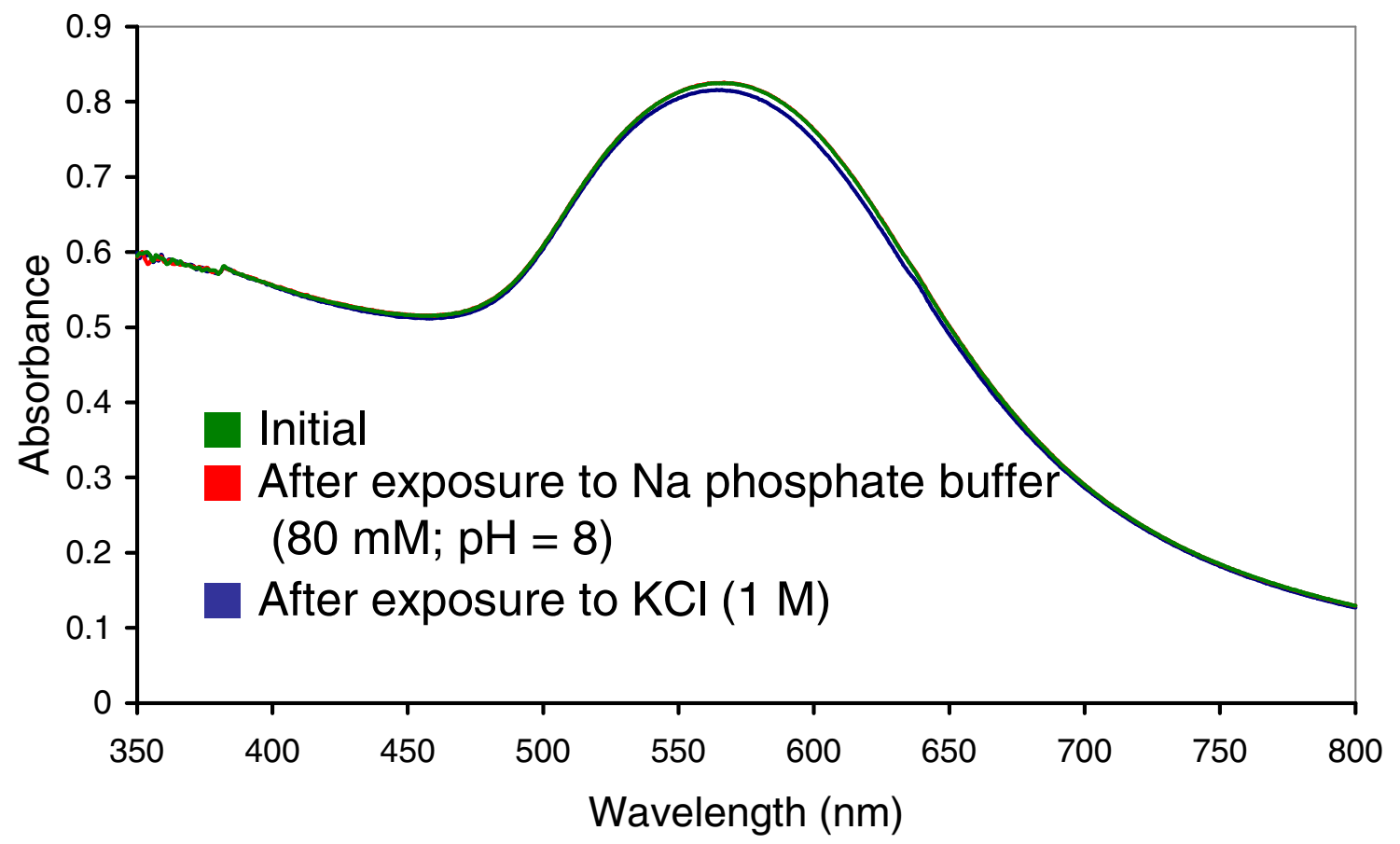

Figure SM-7. PL-linked TAS-NP films before/after exposure to high ionic strength $(\mu)$ solutions of 35 $\mathrm{mM}$ sodium phosphate buffer $(\mu=80 \mathrm{mM} ; \mathrm{pH}=8)$ and $1 \mathrm{M} \mathrm{KCl}(\mu=1 \mathrm{M})$ for 5-10 minutes. Note: Films were assembled as described in the Experimental Details, rinsed with purified water, exposed to solutions of different $\mu$ for the designated time and rinsed with purified water before final immersion in purified water for UV-Vis spectroscopy.

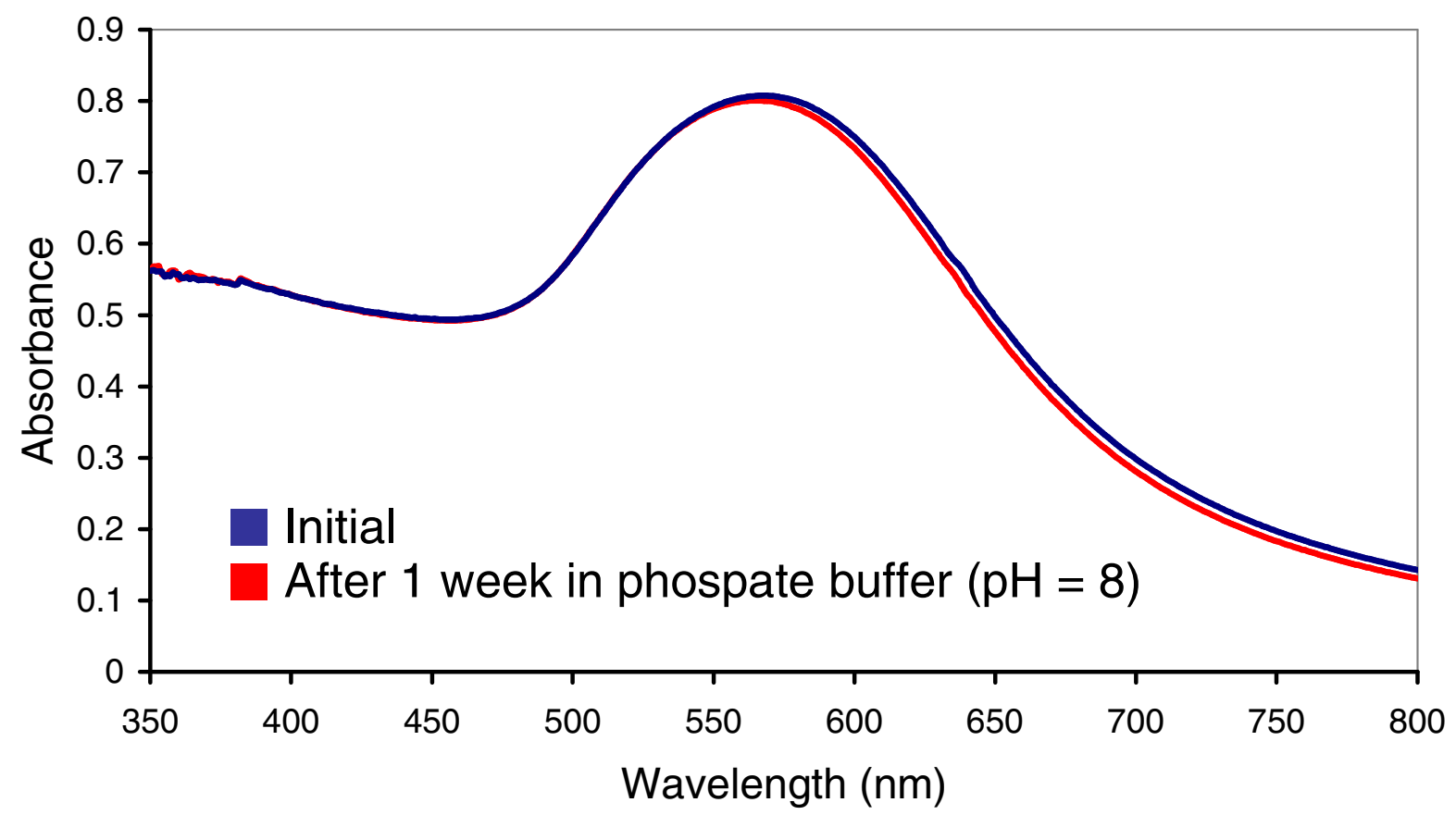

Figure SM-8. PL-linked TAS-NP films before/after exposure to $8.8 \mathrm{mM}$ sodium phosphate buffer $(\mu=$ $20 \mathrm{mM} ; \mathrm{pH}=8$ ) for over one week. Note: Films were assembled as described in the Experimental Details, rinsed with purified water, exposed to solutions of different $\mathrm{pH}$ for the designated time and rinsed with purified water before final immersion in purified water for UV-Vis spectroscopy. 

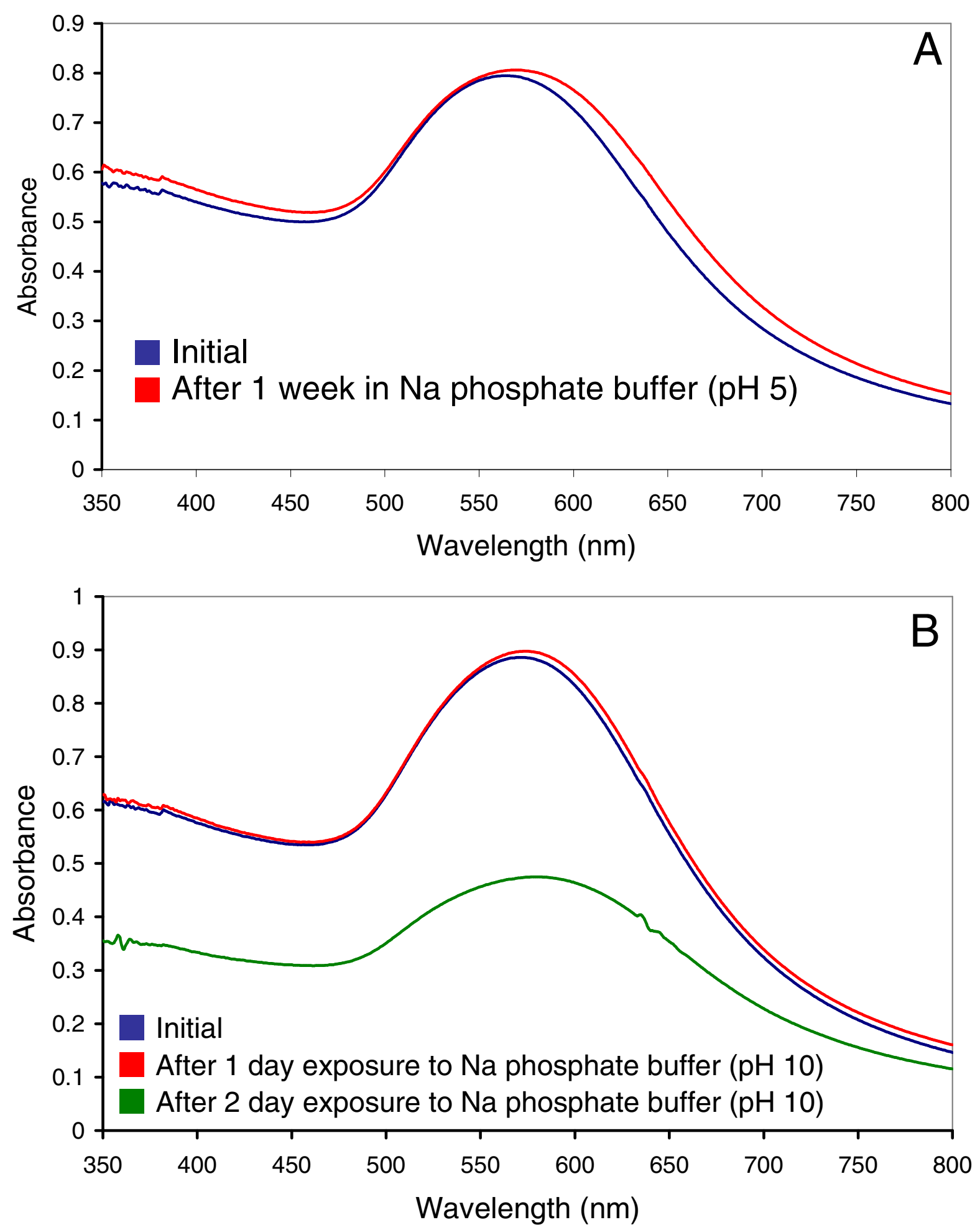

Figure SM-9. PL-linked TAS-NP films before/after exposure to $8.8 \mathrm{mM}$ sodium phosphate buffer $(\mu=$ $20 \mathrm{mM}$ ) at (A) $\mathrm{pH}$ of 5 and (B) $\mathrm{pH}$ of 10 over time. Note: Films were assembled as described in the Experimental Details, rinsed with purified water, exposed to solutions of different $\mathrm{pH}$ for the designated time and rinsed with purified water before final immersion in purified water for UV-Vis spectroscopy. 


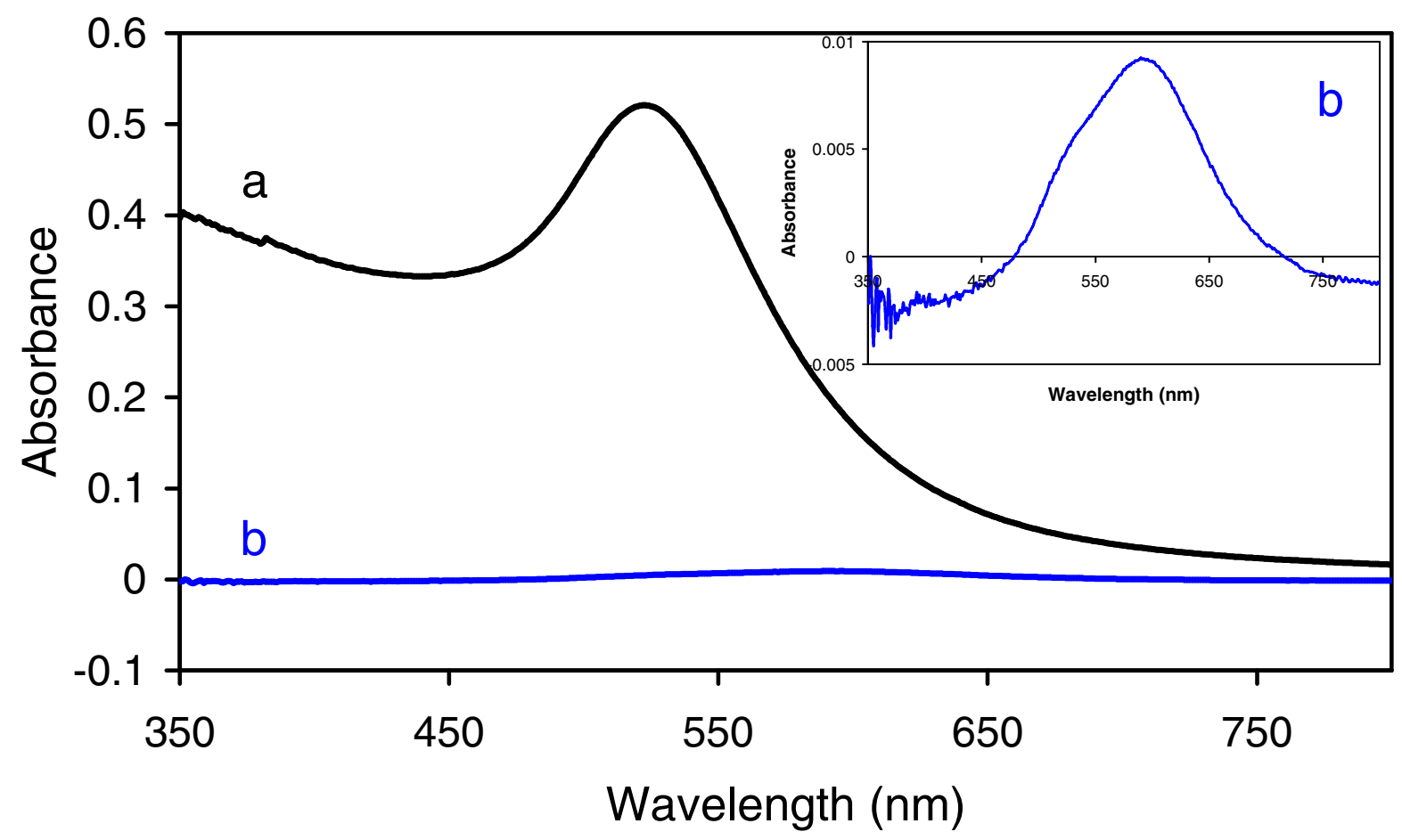

Figure SM-10. Spectra of TAS-NP solution (a) and CS-NP solution (b) after the addition of $0.07 \mathrm{~g}$ of $\mathrm{NaCl}$ to each. The spectrum of the TAS-NPs indicates no change upon addition of $\mathrm{NaCl}$ while the spectrum of the CS-NP solution displays a virtual disappearance of any NP signal, an indication that the NPs have precipitated out of solution via aggregation. ${ }^{\mathrm{b}}$ Inset: Expansion of spectrum (b) showing that the minimal signal remaining after addition of $\mathrm{NaCl}$ to the CS-NPs is characteristic of aggregated NPs (red-shifted from $520 \mathrm{~nm}$ to $\sim 600 \mathrm{~nm}$ ). This result helps to confirm the success of the TA-stabilization process in that a substantial shift in stability (resistance to aggregation) is evident after TA is introduced.

${ }^{\mathrm{b}}$ Cumberland, S.L. and Strouse, G.F. Langmuir 2002, 18, 269-276. 